\title{
La dimensión relacional del gobierno abierto y el liderazgo colaborativo
}

\section{The relational dimension of open government and collaborative leadership}

doi: http://dx.doi.org/10.32870/

espiral.v23i65.4455

\section{Resumen}

En este artículo se explica la dimensión relacional del gobierno abierto, que implica innovación gubernamental sustentada en saberes cívicos, construcción de políticas públicas en red y visión de la gobernanza como nueva forma de gobernar. Se parte de un análisis teórico-conceptual del gobierno abierto y el liderazgo colaborativo, respondiendo a las preguntas: ¿qué rasgos y competencias debe tener un líder público gubernamental en contextos de gobiernos abiertos-relacionales? y ¿con qué saberes cívicos contarían los funcionarios públicos en su relación con los actores no gubernamentales? Se concluye que la gestión pública está hecha del compromiso de los funcionarios públicos y ciudadanos con el Gobierno, facilitando la inteligencia cívica, el control de la gestión vía la contraloría social, así como una concepción compartida de lo público, y que las competencias de la colaboración, lo interactivo y el emprendedurismo son hoy fundamentales para la gestión pública.

Palabras clave: gobierno abierto relacional, innovación en la gestión, liderazgo, colaboración, saberes cívicos.

\author{
Freddy Mariñez Navarro*
}

Keywords: Open-relational government, management innovation, leadership, collaboration, civic knowledge.

\footnotetext{
- Profesor-Investigador del Instituto Tecnológico y de Estudios Superiores de Monterrey.
} fmarinez@itesm.mx 


\section{Introducción}

Definir el gobierno abierto como una organización pública flexible es parte del debate teórico de este concepto en el contexto académico y práctico. El término "gobierno abierto" nos brinda múltiples definiciones, y en cada una se identifican tendencias con un abordaje teórico muy particular. En este sentido, se han sistematizado las distintas propuestas conceptuales de este tipo de organización pública. Una primera definición que ha tenido mucho eco en el mundo académico y gubernamental es la que aborda el gobierno como resultado del cambio tecnológico, donde la web 2.0 juega un rol fundamental, y cuyo centro de análisis son las tecnologías de la información y la comunicación (TIC) (Nan, 2012; Harrison, 2011; Noveck, 2009 y 2010). Una segunda definición enfatiza la importancia de concebir gobierno abierto como un cambio en los valores de la organización y sus procesos, en síntesis, esta definición se traduce en una nueva cultura organizacional (Calderón y Lorenzo 2010; Ramírez Alujas, 2011). La tercera definición con la que se ha identificado el gobierno abierto es aquella que se explica a través del open data, o "datos abiertos", la cual revoluciona el concepto de transparencia tal como se implementaba (Open Government Partnership, 2011; Fung, Graham y Weil, 2007; Bouhadana y Gilles, 2014; Sharon Dawes, 2010 y 2011). Se ha visualizado una cuarta definición, donde el Gobierno abre la posibilidad de la participación de los ciudadanos para monitorear e influir los procesos de gobierno a través del acceso a la información y el acceso a la "arena" de la hechura de las decisiones y de las políticas (Meijer, Curtin y Hillebrandt, 2012; Guardián Orta, 2010; Calderón y Lorenzo, 2010).

La mayor parte de estas definiciones de gobierno abierto tienden a precisar sólo la dimensión vinculada con la apertura gubernamental, vista en el marco de la transparencia 
y el acceso a la información. En este artículo, se retoma para el gobierno abierto su dimensión relacional, por una parte asumiendo las diferentes relaciones de colaboración entre el Gobierno y los ciudadanos y el sector económico, y las relaciones intra- e intergubernamentales (Mariñez y Valenzuela, 2013); y, por otra parte, conectando esta dimensión con la gestión pública participativa sustentada en saberes cívicos, las políticas públicas con visión de redes y la gobernanza, entendida como el

[...] cambio del proceso, modo y patrón de gobierno, que es bien entendido y formulado como el paso de un centro a un sistema de gobierno en el que se requieren, activan, conjuntan los recursos del poder público, de los mercados y de las redes que crean y enlazan los grupos sociales (Aguilar, 2013a, p. 300).

De este modo, el artículo se abordará a partir de la perspectiva teórica, donde se precisará el concepto de gobierno abierto relacional (GAR) como contexto del liderazgo colaborativo. Los objetivos del trabajo son: 1) analizar la dimensión relacional del concepto de gobierno abierto; 2) determinar las líneas generales de relación entre los componentes del gobierno abierto relacional, como la innovación en la gestión pública basada en los saberes cívicos, la gobernanza y las políticas públicas; y 3) identificar los rasgos característicos del liderazgo colaborativo en el contexto del gobierno abierto relacional (GAR).

\section{Una mirada al gobierno abierto relacional (GAR)}

Definido también como collaborative public management (McGuire, 2009), citizen engagement (Kickle, 2008), wiki government (Noveck, 2009), y public sector clients (Alford, 2009), el "gobierno abierto", con sus distintos enfoques esbozados en la introducción de este artículo, tiende a identificar, 
evaluar y caracterizar diferentes formas de integración ciudadana en el sector público a través del marco de la transparencia y el acceso a la información, así como por la colaboración y la participación. Desde los primeros debates, el gobierno abierto presenta una dimensión relacional expresada por dos elementos: 1) la colaboración a través de las TIC, y 2) la participación. La participación colaborativa se da en el momento en que los individuos -con su expertise- son parte integrante con el Gobierno en el proceso de la hechura de las decisiones, buscando generar soluciones que luego serán implementadas. Esto implica un cambio en el juego de las legitimidades políticas, técnicas y sociales de los actores no gubernamentales, además de transformaciones en las organizaciones públicas presionadas por ambientes cada día más complejos (Rainey, 2003). Tanto en las iniciativas que provienen de la ciudadanía, y que en sus procesos integran a los ciudadanos como coproductores, así como en las relaciones entre el sector privado y el Gobierno por la vía de las asociaciones público-privadas (APP), y las relaciones intragubernamentales, el uso de las TIC digitales posibilita este intercambio de experiencias y conocimientos para la solución de problemas (Mariñez y Valenzuela, 2013). En otras palabras, la colaboración abierta ofrece una posibilidad potencial para construir de forma más eficaz y legítima los Gobiernos relacionales (véase Figura 1).

O'Leary y Vij (2012) afirman que, en las actuales circunstancias, y en el marco del gobierno abierto, se necesitan gerentes públicos que trabajen colaborativamente, facilitando y operando arreglos multiorganizacionales para resolver problemas que no pueden ser resueltos, o fácilmente resueltos, por una sola organización. Lo anteriormente afirmado por O'Leary y Vij (2012) ha sido producto de algunos cambios en los ambientes público y privado, así como en los espacios de las organizaciones de la sociedad 
Figura 1. Dimensiones del gobierno abierto

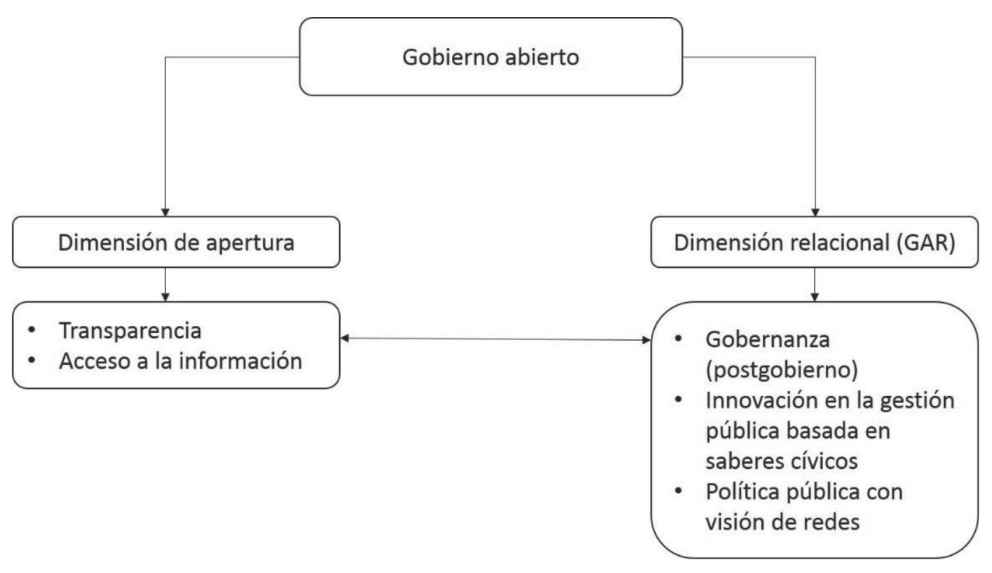

Fuente: elaboración propia.

civil y sin fines de lucro, que a su vez han motivado el crecimiento de lo público colaborativo.

Esta dimensión del gobierno parte de la consideración de que cualquier reto que se plantee una sociedad, bien como gobernanza, gobiernos en redes o innovación con saberes cívicos, es un desafío colectivo que hay que abordar articulando una acción que comprometa a los principales actores y obtenga el máximo apoyo social (Pascual y Tarragona, 2009).

Desde esta definición, un problema adicional se presenta entonces en los gobiernos actuales: el tratamiento racional del control de las burocracias. Hoy vemos que estas están reconfigurándose cada vez más, bajo las presiones de la globalización, la personalización en masa y la hipercompetencia, así como la conversión de las grandes organizaciones en organizaciones flexibles y, en muchos casos, virtuales: "Los formatos postburocráticos se han propuesto, para producir una mayor influencia compartida, el consenso y la confianza en el interior de toda la organización, obviando la necesidad de la jerarquía y la conducta gobernada por 
reglas rígidas" (Sewell, 1998, citado en Raelin, 2011, p. 138). En este sentido, dice Lambright (1971, citado por Pesch, 2008):

Prácticamente todo el mundo admite ahora que la administración pública existe en un entorno político y que el funcionario público debe interactuar con las fuerzas de ese entorno. El funcionario es un participante en el proceso político, un político que debe participar en la resolución de conflictos, en el ejercicio de la discreción y en la toma de decisiones que competen a las demandas (p. 174).

Asumiéndose así, los ocho principios que Siriani (2009) propone en la literatura de la colaboración en la organización pública contemporánea nos llevan a comprender que la función de la política hoy sería: a) habilitar la coproducción ciudadana de los bienes públicos; b) activar la movilización de recursos de la comunidad; c) involucrar la utilización de los conocimientos de expertos en el empoderamiento de los ciudadanos; d) activar la deliberación pública; e) promover alianzas entre ciudadanos, grupos de interés organizados y agencias públicas; f) construir campos y redes de gobernanza estratégica; g) transformar las culturas institucionales para apoyar la práctica de colaboración continua; y h) asegurar la rendición de cuentas recíproca entre la compleja gama de actores.

El Gobierno hoy ha adquirido características que han transformado el concepto cerrado de organización burocrática. Tanto en su estructura organizativa, como en su funcionamiento, así como en su relación con el entorno global, el Gobierno y la administración pública han tomado forma de red, de gobernanza flexible que aborda exitosamente el desafío de construir y acreditar, en los próximos años, la capacidad directiva de los Gobiernos democráticos, a condición, por un lado, de que sea capaz de sustentar la formulación y decisión de las políticas públicas con rigor 
analítico y proceder conforme a criterios rigurosos de eficiencia económica y calidad en la provisión de los varios servicios públicos y, por otro lado, de que sea respetuosa de los resultados que arroja la deliberación sobre los asuntos públicos, impulsada por los ciudadanos en interlocución con los poderes públicos (Aguilar, 2013a). En este sentido, Kelly y Dodds (2012) argumentan que

[...] la administración pública moderna ha incorporado muchas de las inquietudes de la nueva gestión pública. Ahora incluye, directa $\circ$ indirectamente, la consideración de la promoción de valores como la transparencia, la rendición de cuentas, la eficacia y la eficiencia; la movilización y/o incentivo del personal, las habilidades necesarias para una gobernanza eficaz; $y$ en términos generales, las capacidades de las organizaciones para alcanzar sus objetivos de política (p. 204).

\section{Innovar la innovación: cambios relacionales entre el Gobierno y los ciudadanos}

Para los efectos de este trabajo, la innovación en la gestión pública en el GAR es concebida como el reconocimiento de la creatividad y el resultado que imprimen los ciudadanos y actores no gubernamentales en su relación con los funcionarios públicos. Es decir, la dimensión del GAR es fundamental en este enfoque para diferenciarlo del enfoque clásico de la innovación en la gestión sustentada sólo en los procesos y en el uso -como un fin- de las herramientas tecnológicas (Mulgan y Albury, 2003; Windrum, 2008). La innovación constituye, de esta manera, la implementación de un proceso de mejora, o nuevo de gestión, relativo a tres ámbitos: 1) el producto, bien o servicio (introducción de un nuevo servicio o una mejora de la calidad en un servicio existente); 2) el proceso operativo (alteraciones o cambios en las formas de proveer servicios públicos); y 3) la organización o proceso 
de gestión (cambios en las estructuras organizativas, en las prácticas de gestión y en las rutinas) (OCDE, 2012). En este sentido, la eficacia y la eficiencia de las organizaciones públicas se han buscado a través de la mejora de la calidad y de la innovación gubernamental sustentada en los pilares de la calidad total: la satisfacción de los clientes, la mejora continua de los procesos y el desarrollo del personal.

La aplicación de este concepto dio como resultado el reacomodo del arreglo tradicional de las organizaciones, induciendo cambios como el privilegio de los resultados financieros de las empresas y de los Gobiernos, desplazándose hacia la calidad de los productos y enfatizándose, en el sector público, en la eficiencia económica de las operaciones y el equilibrio riguroso de las finanzas públicas; también hizo énfasis en la productividad, moviendo la atención de los productos hacia los clientes, generando que el producto sea un componente integrado del servicio, elevándose la productividad a la dimensión de competitividad por la calidad de los productos. Por otro lado, vemos que la administración hacia adentro, que caracterizó a la administración weberiana, se ha extrovertido hacia los usuarios de los bienes y servicios de la organización, poniendo el punto final de una administración pública sin público y un Gobierno sin ciudadanos (Aguilar, 2006).

El GAR se concibe entonces como el conjunto de innovaciones que tienen por objeto la participación y la colaboración para dar cabida a nuevas formas de articulación entre la sociedad y el Gobierno.

\section{Los saberes cívicos para innovar la innovación}

en la gestión del GAR

En este apartado del artículo, se incorpora el concepto de saberes cívicos para explicar la innovación en la gestión pública. En este sentido, estos saberes están hechos a partir del compromiso de los funcionarios públicos y ciudadanos 
con el Gobierno, facilitando de esta manera la inteligencia cívica, el mejoramiento del control de la gestión vía la contraloría social, así como una concepción compartida de lo público -entendida como un conjunto de competencias de colaboración y cocreación para el logro legitimado de la eficiencia y la eficacia de los gobiernos-.

Los saberes cívicos en la innovación de la gestión en el GAR se convierten en los aspectos sustantivos de la innovación misma. Para entenderlo con más precisión se requiere de la definición de sus cuatro componentes. El primero de los saberes cívicos es el compromiso cívico. Este se entiende como la forma en que los ciudadanos, a través de una obligación o promesa, logran impulsar el asociacionismo con la idea central de llevar a cabo el debate, la deliberación y la solución de problemas públicos. Se trata de un punto más allá de la participación ciudadana que asegura que toda la gente haga uso de sus derechos de libertad, ya que estos y la ciudadanía, según Barber (2004), son correlativos: cada uno sostiene y da vida al otro. No importa la seguridad, los derechos individuales y la autonomía que disfruten: los hombres y mujeres que no se hacen directamente responsables de las políticas que determinan sus vidas, a través de la deliberación, la decisión y la acción comunes, no son en absoluto libres. En este orden, Subirats (2015) nos precisa que

[...] los debates sobre la gestión de recursos, la gestión de servicios, el gobierno y la gestión de las instituciones, toman otro sentido si se parte de las siguientes premisas: construir una nueva política local desde los ciudadanos, desde los barrios, desde los expertos y saberes ciudadanos, desde los políticos-ciudadanos, desde los que saben por qué $y$ han padecido y luchado contra los efectos de las decisiones que han ido tomando los que aseguraban que sabían lo que hacían y que lo hacían por nuestro bien (p. 2). 
Nos referimos entonces a las diversas formas de diálogo ciudadano que son altamente inclusivas y a la deliberación para la hechura de las políticas y decisiones públicas. Así, "la clave de la virtud deliberativa como la reciprocidad, no es más que argumentar en términos que otros pueden aceptar" (Gutmann y Thomson, 1996, citado en Dryzek, 2009, p. 1382). La inclusión es un aspecto importante cuando hablamos del compromiso cívico, ya que tiene como objetivo proporcionar a las personas un sentido de dirección para que las actividades asociativas y comunitarias tengan voz en su relación con el Gobierno, lo que le ayude a mejorar sus decisiones. El éxito del compromiso cívico con el Gobierno requiere de innovación (Fung, 2008).

Así, pues, el compromiso cívico ciudadano le da soporte efectivo al funcionamiento de la democracia, al Gobierno y a la administración pública, generando contextos para la legitimidad del Gobierno, el éxito de la implementación de la política pública y el logro de resultados sociales, es decir, valor social y valor público.

Compromiso ciudadano, para nuestra definición, significa algo más que la participación ciudadana. La diferencia radica en el modelo de producción de governance. Participación ciudadana se da en un modelo de producción tradicional de gobierno donde los ciudadanos son actores o partes interesadas que actúan en un papel de consulta para y con las instituciones establecidas. Compromiso ciudadano sale de un modelo de coproducción de la gobernanza donde los ciudadanos son una parte esencial del proceso de producción. Ciudadanos comprometidos están, en teoría, comprometidos con algún sentido más amplio del "bien común”, más allá de sus intereses individuales (King y Martinelli, 2005, p. 2).

La visualización de King y Martinelli (2005) es pertinente, ya que el compromiso de los actores gubernamentales y no gubernamentales, y a nivel organizativo en sus asociaciones

\section{6}


en la vida pública, es una amplia tendencia en los Gobiernos y administraciones públicas. Esto pone el rol de los funcionarios públicos en este compromiso en el centro del debate. Hoy vemos en esta tendencia el paso de un funcionario público experto-neutral a un facilitador de la participación y el compromiso: "En este escenario, los administradores públicos crean oportunidades para comprometerse con los ciudadanos y utilizar esas oportunidades para educar, organizar y activar a los ciudadanos para defender y participar en una esfera pública más amplia" (Brainard y McNutt, 2010, p. 841).

De este modo, el compromiso cívico también se expresa como un proceso de comunicación directa, tanto presencial como a través de las tecnologías de la información disponibles, y mediante el cual la gente discute de manera abierta sus razones, intereses, habilidades y valores, con la intención de llegar a un acuerdo para la toma de decisión: "[El] compromiso cívico ciudadano en las redes sociales permite a los individuos expresar sus intereses y demandas al Gobierno. También permite que las voces de los individuos sean escuchadas, conduciendo a una mayor inclusión. Las redes de compromiso cívico también hacen a los ciudadanos más competentes" (Michels, 2011, p. 278).

King y Martinelli (2005) afirman que el empoderamiento y el compromiso de los ciudadanos deben ir de la mano de las siguientes "interfaces":

- La "interfaz" entre el político y los ciudadanos (políticos), que incluye la efectividad de las técnicas y las herramientas.

- La "interfaz" ciudadano-burocracia (administración), que incluye el compromiso ciudadano y el empoderamiento en el desarrollo de las políticas públicas y en el establecimiento de prioridades.

- La rendición gubernamental de cuentas, que incluye el compromiso ciudadano para mejorar los rendimientos 
del Gobierno, así como la rendición de cuentas para mejorar los procesos y resultados.

También Bailur y Gigler (2014), parten de que el compromiso cívico implica el empoderamiento ciudadano, para lo cual se requieren los recursos de la participación, la transparencia y la rendición de cuentas. Por un lado, la participación hace elevar a los ciudadanos sus requerimientos y voces (con la idea de que sean escuchados). En este sentido, las herramientas web 2.0 proveen una oportunidad para empoderar a los ciudadanos porque aminoran los impedimentos para participar. Ellos pueden acceder con estas herramientas directamente a la información y comunicación, en lugar de ser dependientes de actores intermediarios.

Por otro lado, la transparencia es un recurso importante para el empoderamiento porque se trata de cualquier intento (del Gobierno o los ciudadanos) por colocar información o procesos que antes eran opacos en el dominio público para su uso por parte de los grupos ciudadanos, los proveedores o los hacedores de política.

El tercer recurso del empoderamiento es la rendición de cuentas (accountability), entendida como la relación entre el poder titular (proveedor titular) y el poder delegado (los demandantes). Para adquirir información sobre acciones y su pertinencia, elaboración de las decisiones e identificación y sanción de resultados insatisfechos, se requiere de las normas establecidas (véase Figura 2). Heeks (2000, citado en Bailur y Gigler, 2014) asume que las TIC permiten que el empoderamiento esté basado en las condiciones siguientes: a) la disponibilidad y transparencia de los datos; b) su accesibilidad para los hacedores de políticas públicas, quienes son capaces de evaluar y transformar los datos en información; c) la información, que es usada para iniciar el dialogo y el activismo entre ciudadanos-Gobierno y

\section{8}


ciudadanos-ciudadanos; y d) el Gobierno, que toma acciones con base en estos procesos.

Figura 2. Visión del empoderamiento

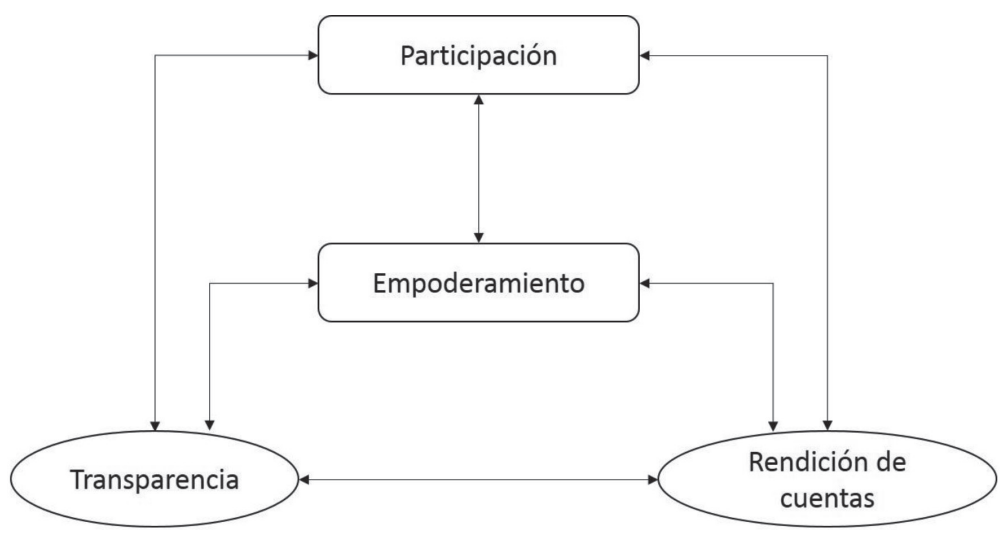

Fuente: Bailur y Gigler (2014).

Es visible, entonces, que la inclusión es un aspecto importante cuando hablamos de participación deliberativa y de compromiso cívico, ya que tiene como objetivo proporcionar a las personas un sentido de dirección a las actividades asociativas y comunitarias para que tengan voz en su relación con el Gobierno, lo que ayude a este a mejorar sus decisiones. ${ }^{1}$ Visualizar el compromiso de los ciudadanos individuales y a nivel organizativo en sus asociaciones en la vida pública es una amplia tendencia en la gestión pública y una base fundamental del gobierno abierto relacional.

I. En esta dirección, Fung y Wright desarrollaron la visión del gobierno participativo con poder de decisión (GPPD), sustentado en los principios: a) concentración en problemas específicos y tangibles (orientación práctica); b) participación activa de la gente común y corriente a quien afectan esos problemas y de los funcionarios cercanos a ella (participación desde la base); y c) desarrollo deliberativo de soluciones a esos problemas (generación de discusiones deliberativas) (Fung y Wright, 2010, p. 156). 
El otro componente de los saberes cívicos es la inteligencia cívica, vista como la inteligencia colectiva directa, dirigida a enfrentar los desafíos compartidos. Es decir, dirigir las capacidades percibidas, actuales y potenciales de los individuos, grupos y organizaciones sociales, para responder inteligente, efectiva y equitativamente a los desafíos actuales con miras a lograr el desarrollo de la convivencia democrática. Su presencia o ausencia determinará cómo efectivamente estos desafíos pueden enfrentarse.

A causa de que el Gobierno y otras élites grupales no son capaces de hacer frente a los problemas, se requerirá una forma más profunda de inteligencia cívica construida por ricas interacciones entre ciudadanos distribuidos en todo el mundo. Esta inteligencia no emergerá únicamente de una serie de votos u otras técnicas algorítmicas (Schuler, 2010, p. 92).

El mismo Schuler (2010) presenta una definición cuyo punto de partida son los diversos tipos de proyectos ciudadanos y comunitarios para motivar y coordinar diversos esfuerzos. Esto refiere a cómo la inteligencia cívica está resolviendo sus problemas comunes y qué grupos de personas persiguen fines cívicos a través de medios cívicos. Además, este autor precisa el concepto de inteligencia cívica como la "capacidad de los grupos y organizaciones, e idealmente la sociedad en su conjunto, para concebir y poner en práctica estrategias eficaces, equitativas y sostenibles para compartir problemas" (Shuler, 2008, p. 81). Sin duda, como ejemplo de innovación social, es una respuesta a una necesidad percibida, en la cual la recepción que tiene, o no, por parte de los ciudadanos, va en proporción a la necesidad de percepción que estos tengan. Siguiendo esta misma línea argumental, Shuler (2008) describe un modelo de inteligencia cívica que contiene los seis aspectos que se señalan en el Cuadro 1.

Para complementar este modelo descriptivo, Schuler (2008) propone un modelo funcional que precisa cómo 
Cuadro 1. Modelo descriptivo de la inteligencia cívica

\begin{tabular}{ll}
\hline Orientación & $\begin{array}{l}\text { Fines, principios y perspectivas que ayudan a energizar el } \\
\text { despliegue efectivo de la inteligencia cívica. }\end{array}$ \\
Organización & $\begin{array}{l}\text { Estructuras, métodos y roles por los cuales la gente com- } \\
\text { promete su inteligencia cívica. }\end{array}$ \\
Compromiso & $\begin{array}{l}\text { Formas en que la inteligencia cívica se convierte en un } \\
\text { activo y en una fuerza de provocación para el pensa- } \\
\text { miento, la acción y el cambio social. }\end{array}$ \\
Inteligencia & $\begin{array}{l}\text { Formas en que la inteligencia ciudadana se manifiesta a } \\
\text { través del aprendizaje, la formulación e intercambio de }\end{array}$ \\
& $\begin{array}{l}\text { conocimientos, la interpretación, la planificación, la meta- } \\
\text { cognición, etc. }\end{array}$ \\
productos y & $\begin{array}{l}\text { Algunas de las formas, tanto a corto como a largo plazo, } \\
\text { en que los organismos de inteligencia civiles centran sus }\end{array}$ \\
& esfuerzos; incluye resultados tangibles y campañas para \\
ayudar a alcanzar los objetivos deseados.
\end{tabular}

Fuente: Shuler (2008, p. 87).

debe implementarse la inteligencia cívica. Este modelo contiene tres componentes principales: el medioambiente, que incluye todo lo que es relevante para la organización, pero que está fuera de la organización; el modelo básico (o núcleo), que corresponde a la suma del conocimiento que la organización utiliza; y los componentes de la organización, que incluyen sus recursos (por ejemplo, personas) y, lo más importante, los procesos interactivos en el marco del control de la organización que vincula el medioambiente con el modelo mental. El modelo funcional contiene ocho tipos de procesos interactivos que las organizaciones exhiben cuando realizan la inteligencia cívica: 1) monitoreo; 2) discusión y deliberación; 3) compromisos; 4) transferencia de recursos (estos cuatro primeros se inscriben en el componente organizacional que interactúa con el medioambiente); 5) 


\section{interpretación de nueva información; 6) mantenimiento del modelo básico; 7) planificación y plan de acción; y 8) modi- ficación del modelo básico (estos últimos cuatro procesos pertenecen al componente básico, que también interactúa con el medioambiente):}

\section{Cuadro 2. Modelo funcional de la inteligencia cívica}

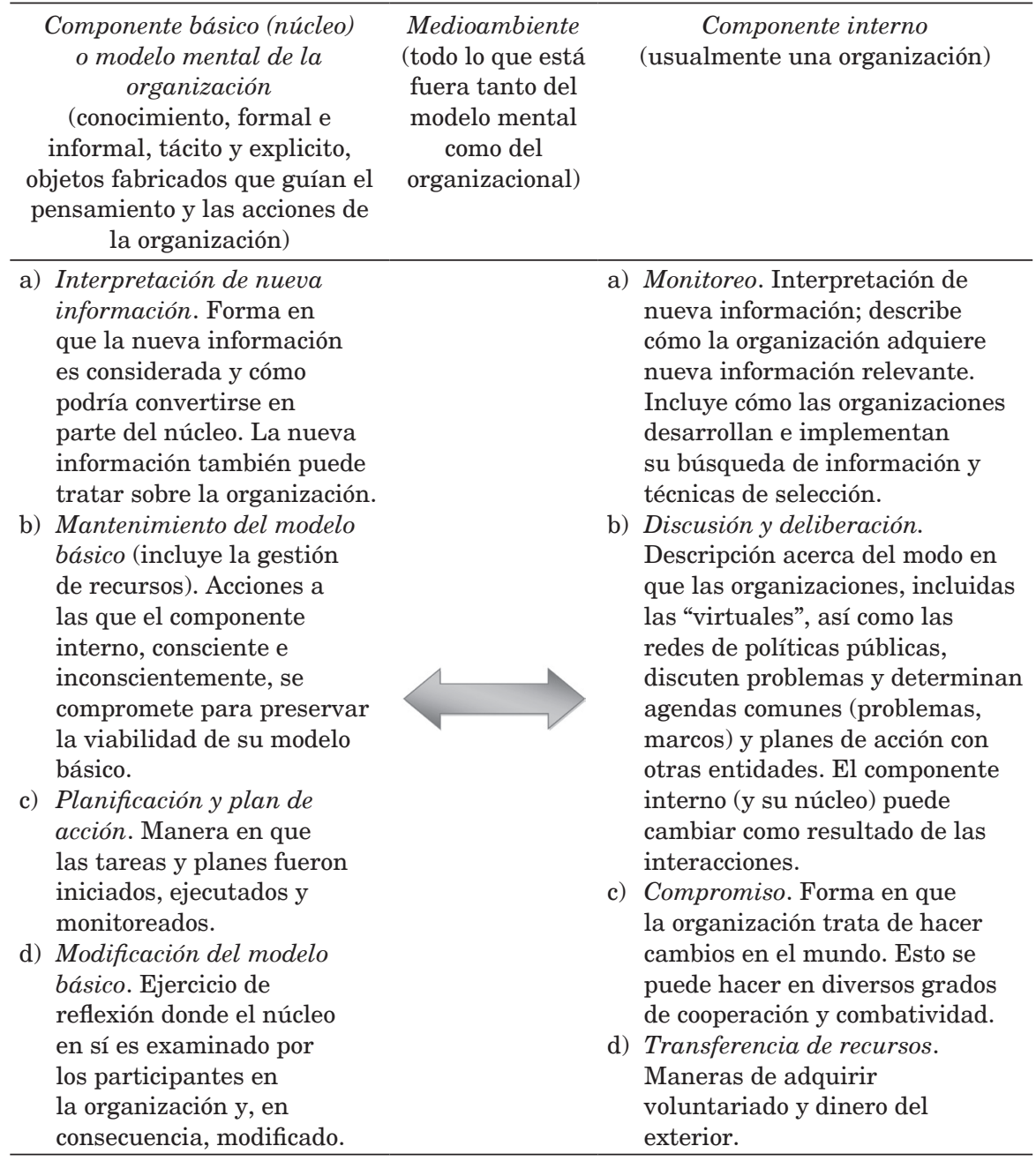

Fuente: Shuler (2008, pp. 87-88). 
La inteligencia cívica no es más que tener información y comprenderla para compartirla a quienes necesitan respuestas. En otras palabras, implica conocer, responder y proveer, por lo que se podría entender como la capacidad de las organizaciones ciudadanas y comunitarias de adquirir y aplicar conocimientos, o bien, como la capacidad para adaptar, efectiva o simplemente, aprendizaje, razonamiento, predicción, reflexión e imaginación de los ciudadanos para la resolución de problemas (LaDuke, 2008).

El modelo funcional de la inteligencia cívica se centra en el rol de la sociedad civil y lo público, y su carácter es participativo y colaborativo. Visto así, no es más que la lógica de la cocreación de soluciones, definición de problemas sociales y de colaboración. Por eso, el crowdsourcing ${ }^{2}$ juega un papel importante en la inteligencia cívica, ya que los ciudadanos aprovechan el internet para adquirir recursos, conocimiento, experiencia o tiempo (colaboración abierta). Zhao y Zhu (2012, citados en Seltzer y Mahmoudi, 2012) definen el crowdsourcing como un sistema de inteligencia colectiva, y Surowiecki (2005) habla de la sabiduría del público: "en las circunstancias adecuadas, los grupos son muy inteligentes, y con frecuencia son más inteligentes que las personas más inteligentes del grupo" (Surowiecki, 2005, citado en Seltzer y Mahmoudi, 2012, p. xiii).

El tercer componente de los saberes cívicos es el control social, que es la participación ciudadana orientada al monitoreo, la vigilancia y la evaluación por parte de personas u organizaciones sobre programas y acciones gubernamentales, incidiendo directamente en la esfera pública, ya sea formalmente-por medio de su inclusión en comités de obras, órganos colegiados, etc.-, o indirectamente-por medio de la

2. Entendido como la apertura de los problemas a la colectividad, con las ventajas de compilar una gran variedad de propuestas de alta calidad por parte de la comunidad y generar en forma continua ideas innovadoras a través de un feedback interno y permanente. 
activación de los sistemas de quejas y denuncias- (Hevia de la Jara, 2006). Lo cierto es que el control social, en vez de originarse desde las instituciones administrativas y políticas, emana desde la sociedad: es el control directo desde los ciudadanos a los políticos y funcionarios públicos.

Hoy se tiene un nuevo marco de análisis para el control social a partir de las tecnologías web 2.0. A través del software social, se logran una serie de aplicaciones que pretenden ampliar las posibilidades de comunicación y de conexión entre personas, ya que permiten el e-mail o los sistemas de mensajería instantánea. Son herramientas colaborativas que unen a una serie de personas con el fin de facilitar el flujo de información (agendas, mensajes, denuncias) y la realización de trabajos conjuntos entre ellas (colaboración en el monitoreo o vigilancia de las políticas públicas), produciendo lo que Curtin y Mendes (2012, citados por Meijer, Curtin y Hillebrandt, 2012) denominan voz y visión: "los ciudadanos necesitan información para ver qué se está haciendo al interior del Gobierno, y participación para expresar sus opiniones sobre el quehacer gubernamental" (p. 11). De esta manera, los ciudadanos no solamente tienen acceso a la información, sino que se convierten en participantes con expresión activa.

Para el logro de la voz y visión en el control social, se parte de cuatro ejes que hacen a estos más efectivos:

a) Primer eje: transparencia colaborativa y relacional. Debe existir un proyecto de datos abiertos, aplicado como una filosofía y forma de administración, donde todos los datos públicos estén disponibles y actualizados en un formato libre, gratuito y sin ninguna restricción. No sólo se debe ofrecer transparencia a la gestión, sino acceso a datos comunes y necesarios que pudieran ser utilizados por toda la población para diversos fines, como información de índole económica, estadística, meteorológica, geográfica o turística, así como social, la salud y la educación, 
así como series de datos que no pueden ser obtenidos fácilmente. Además, se debe alentar la colaboración, el trabajo conjunto de las organizaciones o particulares con los datos oficiales y la participación de las personas como auditores implícitos de la gestión.

b) El segundo eje se relaciona con la organización y las capacidades de los ciudadanos. Precisar la organización y la capacidad ciudadana online para el control social; se requiere que los ciudadanos logren la legitimidad técnica para poder hacer uso de las plataformas de software social y sus herramientas de colaboración, que permiten comunicar, coordinar y compartir recursos, aplicaciones y contenidos. Pero, ¿cómo pueden permitir las TIC el empoderamiento de los ciudadanos? Por una parte, con los flujos de información de arriba hacia abajo: desde el Gobierno a los ciudadanos. Por la otra, con la creación de la posibilidad de flujos de abajo hacia arriba: de los ciudadanos hacia el Gobierno, que son esenciales para la toma de decisiones. Y por último, los flujos de comunicación horizontales, tales como las redes interorganizacionales y las redes sociales. En este sentido, se habla de un control social en red y no jerárquico.

c) El tercer eje es el acceso a la información. Se parte de la base de que el acceso a la información es un derecho ciudadano, reconocido en muchos países a nivel constitucional. Como derecho fundamental, está enmarcado en la Declaración Universal de los Derechos Humanos adoptada por la Asamblea General de la Organización de las Naciones Unidas (ONU) en su artículo 19: "Todo individuo tiene derecho a la libertad de opinión y de expresión; este derecho incluye el de no ser molestado a causa de sus opiniones, el de investigar y recibir informaciones y opiniones, y el de difundirlas, sin limitación de fronteras, por cualquier medio de expresión" (ONU, s/f). En el caso mexicano, el derecho de acceso a la información pública se 
encuentra establecido como una garantía individual en el artículo $6^{\circ}$ de la Constitución Política de los Estados Unidos Mexicanos, y está reglamentado en la Ley General de Transparencia y Acceso a la Información Pública, publicada el 16 de abril de $2015 .^{3}$

d) El cuarto eje se relaciona con lo público. El control social no sólo se aplica al Gobierno, sino a todos los actores que intervienen en la producción del valor público. Saxton y Guo (2011) han venido afirmando, con base en sus investigaciones, que con la difusión de las tecnologías del internet aparecen dos dimensiones fundamentales de la rendición de cuentas de las organizaciones civiles: la divulgación y el diálogo. Esto quiere decir que si la producción de lo público es colaborativa (cocreación), los diversos actores (gubernamentales y no gubernamentales) son objeto de ser controlados por sus acciones.

De una manera más precisa, el control social es el medio para ejercer el monitoreo, la fiscalización y la vigilancia de la ejecución del plan de desarrollo de la política y el servicio públicos, por una parte, permitiendo prevenir a tiempo a los actores de la cocreación pública sobre irregularidades, desviaciones de los recursos destinados a obras, servicios, programas y proyectos, y sugiriéndoles cómo corregirlas; por otra parte, contribuyendo al cumplimiento de las leyes y procedimientos en la ejecución de las obras, servicios, programas y proyectos financiados con fondos públicos; y, por último, incentivando la confianza ciudadana en la incorporación social de la vigilancia sobre los recursos destinados a satisfacer las necesidades de la colectividad. "Además, el control social surge como una forma sustancialmente

3. En el marco de esta ley, se establece la creación la Comisión Permanente de Gobierno Abierto y Transparencia (2015), que ha venido proponiendo un modelo de gobierno abierto con estos componentes:acceso a la información, transparencia proactiva, rendición de cuentas, participación ciudadana, cocreación e innovación.

\section{6}


diferente de los modelos tradicionales de control: político, fiscal, judicial, interno, disciplinario, pero, igualmente, es un escenario de interacción entre el Estado y la sociedad civil" (Garcés Lloreda, 2006, p. 480).

El control social no es posible sin el compromiso cívico, la inteligencia cívica y la participación colaborativa, ya que sus objetivos están orientados a garantizar a los ciudadanos el acceso a la información, impulsar la participación y la corresponsabilidad, mejorar la eficiencia, eficacia y confiabilidad de las acciones gubernamentales, e incorporar a la ciudadanía en la vigilancia de la corrupción.

El cuarto y último componente de los saberes cívicos es la participación colaborativa. Es evidente que, para que la participación colaborativa se cristalice y se convierta en un factor de innovación en la gestión de un Gobierno abierto relacional, es menester la existencia de una política gubernamental que posibilite un mayor acercamiento, a través del diálogo, entre Gobierno y ciudadanos, dándosele cabida a la atención y gestión de las demandas, a la negociación, colaboración e interlocución entre ambos. La participación colaborativa difiere de las formas clásicas de participación por la desconexión con el proceso de elaboración de las tomas de decisiones. Harrison, Santiago Guerrero, Burke y Cook (2011) afirman que la deliberación y participación deben dar la oportunidad para el intercambio de visiones con el objeto de determinar el curso de la acción; en otras palabras, deben asegurar que diversos puntos de vista de ciudadanos, grupos civiles y actores no gubernamentales sean incorporados a la acción del Gobierno. Esto significa que la participación colaborativa se da en el momento en que los individuos, con su expertise, son parte integrante, junto con el Gobierno, del proceso de la hechura de decisiones que busquen generar soluciones que luego sean implementadas. Lo anterior nos lleva a ver la lógica gubernamental que opera con arreglos multiorganizacionales para la solución de problemas públi- 
cos, o sea, una forma nueva de relación entre la sociedad y el Gobierno.

Una herramienta importante a considerar en la participación colaborativa son las tecnologías de la información y la comunicación (TIC), mejor conocidas como web $2.0,{ }^{4}$ que demuestran que la gente está bien informada de todo y que, cuando se le da la oportunidad de reunirse en una red y en grupos, puede ser eficaz en la solución de problemas y no sólo en opinar sobre los mismos. Esta afirmación nos deja claro lo que Noveck (2010) ha planteado: la colaboración es una forma de participación democrática que es igualitaria, pero diferente respecto a la concepción tradicional del término. Además, son los cambios generados por los procesos de globalización los que han conformado una sociedad en red, dando como resultado una lógica diferente de la participación basada en la colaboración (Castells, 1999 y 2008).

$\mathrm{El}$ hecho de que la gente esté bien informada la dispone a participar, con sus habilidades, conocimientos, experiencias, información y entusiasmo, en el apoyo a la gestión y política públicas, por lo que una gestión innovadora establecerá los mecanismos e instituciones inteligentes para hacer uso de la tecnología y conectarse con los ciudadanos, haciendo de la colaboración, de este modo, una participación abierta, “que no es más que un tipo de participación pública a través de herramientas de comunicación interactivas como blogs, microblogs o redes sociales. Se basa principalmente en la expresión de medios sociales para conectar a la gente y ayudar a compartir sus ideas" (Cusba, 2012, p. 119).

4. El término web 2.0 fue acuñado en 2005 por Tim O’Reilly, quien definió los siete principios constitutivos de las aplicaciones web 2.0: la web como plataforma, el aprovechamiento de la inteligencia colectiva, la gestión de la base de datos como competencia básica, el fin del ciclo de las actualizaciones de versiones del software, los modelos de programación ligera junto a la búsqueda de la simplicidad, el software no limitado a un solo dispositivo y las experiencias enriquecedoras de los usuarios (O'Reilly, 2005). 
Los saberes cívicos, articulados a la innovación de un Gobierno relacional, responden a dos direcciones: 1) los arreglos de los actores gubernamentales y no gubernamentales que permiten identificar problemáticas públicas, así como herramientas y dispositivos innovadores para el logro de respuestas y soluciones nuevas en el espacio de lo público, y 2) como consecuencia de lo anterior, las modalidades democráticas de articulación de estos actores en la organización pública, que produzcan una nueva configuración entre el Estado y la sociedad que asegure el aprendizaje colectivo y los saberes cívicos necesarios para una gestión inclusiva y de calidad. Sólo así el Gobierno se vuelve más relacional, la gestión pública más participativa y las políticas públicas -en todo su ciclo- son más deliberativas en la construcción de la agenda, más incluyentes en aportar soluciones (diseño) y más colaborativas por los múltiples actores que intervienen en la implementación (Peters, 2014).

En este contexto, esta visión de la innovación de la gestión pública en el marco de un GAR debe ir de la mano con el fortalecimiento tanto de las instituciones democráticas (Rizvi, 2008), como de las relaciones societales y de estas con el Gobierno: "Hay innovación cuando ciertos tipos de problemas no tienen solución en las instituciones existentes. Los actores deben conseguir soluciones innovadoras de sus problemas, dada a la insuficiencia institucional" (Harrison y Klain, 2007, p. 8). Así, resolver esta falencia no hace más que apelar a los saberes cívicos para completar y expandir la confianza en las capacidades de la organización pública, en otras palabras, construir redes de capacidades y de saberes en las que se combinan recursos, inteligencia y expertise de varios actores para la coproducción (Fung, 2002). 
3. Rasgos y competencias del liderazgo colaborativo en el contexto de gobierno abierto relacional

Una visión relacional del gobierno y, por ende, del liderazgo colaborativo ayuda a producir y tomar ventajas de oportunidades para que las organizaciones públicas mejoren su implementación y los ciudadanos construyan ciudadanía con compromiso cívico. Entendido así, se requiere de un emprendimiento público sustentado en saberes cívicos que se convierten en la clave de la organización innovadora. Mothae y Sindane (2013) definen a este emprendimiento como

[...] el desarrollo de nuevos impulsos en el contexto de todo lo amplio de las organizaciones públicas. Funcionarios públicos, a través del emprendimiento, se convierten en emprendedores en sus respectivos sectores de sus organizaciones. Ello implica, así, la necesidad de habilidades tales como las capacidades para resolver problemas creativamente, tomar riesgos calculados, ver funciones $\mathrm{u}$ organizaciones como centro de aprovechamiento, y reconocimiento del lazo entre las funciones organizacionales y los fines a largo plazo (p. II7).

En otras palabras, de lo que se trata es de un nuevo contexto del liderazgo público:

El problema con el paso de la teoría del liderazgo jerárquico a las redes puede descansar diferenciando dos contextos. El entorno de red puede ser más complejo debido a los diversos objetivos que cada miembro de la red tiene por el resultado de la combinación de esfuerzo y a las limitaciones que le son impuestas a la acción colectiva desde las mismas organizaciones de los diversos miembros de la red (Haxham y Vangen, 2000, citados en Silvia, 20I I, p. 68).

De esta manera, el líder de la red debe mantener el apoyo de sus supervisores con el fin de trabajar con éxito fuera de 
sus límites de agencia tradicional. No sólo el líder tiene que convencer a su agencia o dirección principal de la necesidad de llegar a los demás para resolver problemas graves, sino que el mismo líder debe promover la red en otras direcciones y con posibles socios de colaboración para fomentar su participación (Agranoff, 2003). Un contexto institucional para un liderazgo relacional en una organización pública debe sustentarse en la confianza institucional, la transparencia, la disponibilidad de TIC y las competencias de colaboración.

No obstante que la naturaleza del gobierno relacional requiere de funcionarios públicos emprendedores que se preparen para la diversidad, la adaptación y la reinvención, con el objetivo de cumplir las demandas públicas, este presenta problemas en los contextos de gobernanza en lo que concierne al lado administrativo de la gestión de redes.

El problema más frecuente refiere al cambio de los roles de los funcionarios públicos cuando operan asociaciones y redes. Como ellos están acostumbrados a operar organizaciones cerradas y específicas en un modelo de gobierno jerárquico, con límites claros, reglas y procedimientos rígidos, y una amplia autonomía con respecto a la sociedad, los funcionarios públicos carecen de las habilidades y competencias necesarias para la gestión de redes multiorganizacionales, multigubernamentales y multisectoriales (Aguilar, 20I3b, p. 7l).

Aguilar (2013b) también propone acciones prácticas de gobernanza y de innovación pública para emprender un liderazgo en el marco de un gobierno relacional. Tales acciones son el desarrollo institucional para asegurar la participación de los ciudadanos en la hechura de las decisiones (consultas, debates, implementación, control en el gasto y presupuesto y formas de asociación con agencias gubernamentales); la institucionalización de las asociaciones público-privadas y las redes, definiendo su contenido, alcance, redes de acceso, perfil de los miembros y reglas operativas, sin disminuir los 
incentivos en las asociaciones y redes; el aseguramiento de que no solamente los grupos de interés influyentes tengan voz y puedan ser parte de las decisiones públicas, sino también los pobres, la población vulnerable y las personas discriminadas; la promoción de la creación de capital social y construcción de capacidades para mejorar la calidad técnica e institucional de la participación de los ciudadanos; así como la salvaguarda de la transparencia y la rendición de cuentas de los procesos de gobernanza y sus resultados vía la legalidad.

Además, los administradores públicos necesitan estar equipados con una comprensión de cómo los actores que intervienen consideran la conveniencia de interacciones con el Gobierno y el uso de los canales de participación que apoyan la misión de la agencia para lograr los fines. Los métodos e instrumentos fuera de las herramientas de las TIC no apoyan al uso de los medios sociales y tareas: hoy los recursos tienen que ser cambiados para hacer frente a estos desafíos (Mergel, 2012).

Valotti (2012) define las fronteras del liderazgo, en las que establece dos perspectivas: la perspectiva gerencialista, que parte de que todos los aspectos de la vida organizacional pueden ser gerenciados de acuerdo a procesos y estructuras racionales y modos de acountability seguidos por fines definidos por los policymakers y los funcionarios de rango gerencial, y la perspectiva denominada liderismo, que cree que todos los aspectos centrales de la vida organizacional pueden ser coordinados por uno o más individuos que dan dirección o sentido a la actividad social, conducida por sí misma y otros. Este enfoque se basa más en la dimensión relacional y visionaria (véase Cuadro 3). 
Cuadro 3. Enfoques del liderazgo

\begin{tabular}{cc}
\hline \multicolumn{1}{c}{ Visión gerencialista } & Visión liderista \\
\hline 1. Planificación y presupuesto: & 1. Establecimiento de la dirección: \\
establecimiento de agendas & crear una visión, aclarar la \\
y calendarios, así como la & imagen y ajuste de grandes \\
asignación de recursos. & estrategias. \\
2. De la organización del personal: & 2. Alinear a las personas: \\
proporcionar estructuras, & comunicar los objetivos, buscar \\
colocaciones de empleo y el & el compromiso y construir \\
establecimiento de normas. & equipos. \\
3. Control y resolución de & 3. Motivar e inspirar: dar energía, \\
problemas: el desarrollo de & empoderar y satisfacer \\
incentivos, generación de & necesidades. \\
soluciones creativas y toma de & \\
las medidas correctivas. & \\
\hline
\end{tabular}

Fuente: Valotti (2012).

Se ha afirmado ya que hoy la tendencia es el paso de un funcionario público neutral a un facilitador de la participación y el compromiso con habilidades técnicas (Denhardt y Vinzant Denhardt, 2002; King y Stivers, 1998; King y Zanetti, 2005, citados en Brainard y McNutt, 2010). En este escenario, los funcionarios públicos deben crear oportunidades para comprometerse con la sociedad y utilizar esas oportunidades para educar, organizar y activar a los ciudadanos en la defensa de sus derechos, y participación en una esfera pública más amplia. "Los esfuerzos por resolver los problemas públicos complejos y multifacéticos de hoy requieren de acciones colaborativas en muchos lugares y jurisdicciones, utilización de nuevas tecnologías de información y comunicación, y cambio de valores tradicionales" (Agh, 2010, citado en Rosenbaum, 2011, p. 161).

Se podría entender entonces que el liderazgo del funcionario público en la situación actual hace que las instituciones participativas funcionen; un liderazgo que promueva la innovación en el Gobierno, pero también los saberes cívicos, 
a través de la formación de equipos con los actores gubernamentales y no gubernamentales, la generación de redes de trabajo y comunicación, proporcionando retroalimentación, coordinación y manejo de conflictos y cooperación, practicando políticas de colaboración, conformando estrategias para comunicarse con los ciudadanos con la idea de hacer a la organización más transparente y sujeta a rendición de cuentas, es, en otras palabras un liderazgo colaborativo, relacional y que promueve la cocreación a partir de propuestas compartidas.

Además, un liderazgo con estas características tiene que gestionar la diversidad, un fenómeno importante en las organizaciones actuales. Una gestión de esta naturaleza involucra tres componentes que los líderes deben conocer: 1) la atracción y selección de empleados talentosos; 2) la valoración de las diferencias culturales de los empleados, por lo que debería también superar las diferencias culturales (reducción de sesgos intergrupales), generando un valor añadido potencial por el solo hecho de tener diferentes orígenes y puntos de vista; y 3) la necesidad de instrumentos de políticas y programas para la gestión de la diversidad en las prácticas cotidianas (Ashikali y Groeneveld, 2015).

Huxhan y Vangen (2000, citados en Silvia, 2011), identifican tres medios principales a través de los cuales el liderazgo relacional de redes puede ser impulsado: 1) a través de la estructura, donde esta refiere a la organización colaborativa y a los individuos asociados en eso; 2) mediante procesos, es decir, a través de la comunicación colaborativa; y 3) vía los participantes, donde se incluye a los individuos que componen la red, así como a las organizaciones y grupos que ellos representan. A través de estos tres medios, los líderes pueden comprometerse en comportamientos dirigidos a gestionar y controlar la agenda, representar y movilizar a los miembros de la organización y entusiasmar y empode- 
rar a aquellas personas que pueden alcanzar los objetivos de la colaboración.

Con base en Mothae y Sindane (2013), sobre las competencias del liderazgo y la gobernanza en las políticas públicas, se precisa lo que ellos denominan las competencias en la colaboración, en lo interactivo y en el emprendurismo. En cuanto a las competencias de colaboración, son aquellas capacidades que sirven para trabajar más allá de las fronteras organizativas para el logro de resultados. Se pueden identificar las que construyen coaliciones, alianzas y asociaciones. Esto implica una habilidad para identificar, crear, gestionar y sostener estas tres formas de colaboración en busca de la efectiva implementación de la organización y sus políticas. Se alega, además, que las competencias de colaboración ayudan a la confianza mutua, tan necesaria entre las partes interesadas (ciudadanos, funcionarios y sector económico), la transparencia y la rendición de cuentas -que en esencia es parte de una gobernanza colaborativa-.

Por otro lado, el líder público de hoy requiere de competencias de interacción con habilidades para comunicar, participar, establecer redes y manejar las TIC.

Además de estas dos competencias definidas, se identifican las relacionadas con el emprendedurismo, que serían las capacidades relacionales, la visión de las organizaciones del sector público, la habilidad para la toma de cálculo de riesgos y la capacidad creativa para resolver problemas, competencias útiles para llevar a cabo la innovación social.

Por último, se destacan las competencias que todo líder moderno tiene: ser visionario, tener competencias relacionales y competencias personales, como la capacidad de responsabilidad (véase Figura 3). 
Figura 3. Competencias del liderazgo colaborativo

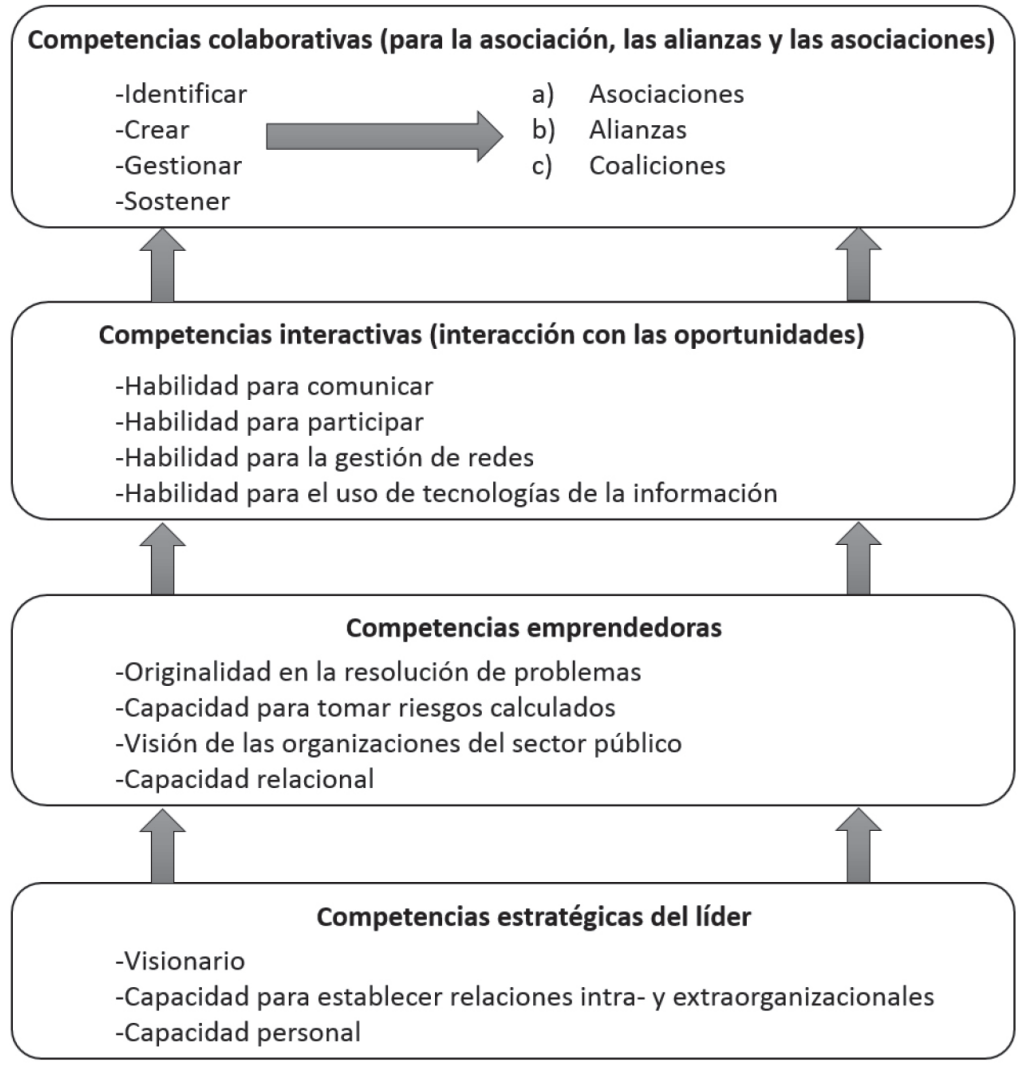

Fuente: elaboración propia con base en Mothae y Sindane (2013).

Es importante destacar que toda organización que viva un proceso organizativo que tienda a la colaboración requiere de la combinación de un liderazgo integrativo y colaborativo con el objetivo de que este facilite a los actores la comunicación y la participación en el diálogo para la cocreación de las decisiones (véase Figura 4).

En un mundo de cambios e incertidumbre, se pone énfasis en la capacidad de comprender la formación y el cambio de los supuestos 
subyacentes del liderazgo tradicional, así como sus mentalidades y sus marcos interpretativos. Esta capacidad es una capacidad preeminente y distintiva de los líderes en el siglo XXI. Hace un llamamiento a los líderes para ejercer un mayor sentido de la reflexividad y para crear las condiciones en las que miembros de la organización pueden reconocer, viendo hacia afuera, los límites ocultos que limitan su pensamiento y comportamiento (McLean, 2013, p. 230).

Se ve, entonces, que el líder colaborativo e innovador aplica ideas y prácticas novedosas en los ámbitos del gobierno relacional y de la gestión y las políticas públicas, generando mejoras en los servicios públicos, colaboración con la ciudadanía y las empresas, así como el mantenimiento de la eficiencia operativa, de manera que no se nubla solamente con las TIC, claves en el proceso de innovación, sino que ve como misión una administración pública que sirva a los intereses generales, cuyos objetivos sean mantener la cohesión social, defender los derechos y libertades de los ciudadanos, y preservar y mejorar la democracia.

Las nuevas habilidades de liderazgo deben incorporar la capacidad para conducir y gestionar el cambio y la innovación, así como para canalizarlos hacia la mejora del valor público. También los nuevos líderes deben ser capaces de comprender las tendencias de la sociedad actual, incluyendo el impacto de las web 2.0 en la relación entre el Gobierno y los ciudadanos, y cómo aprovechar el compromiso de los ciudadanos para mejorar (Bertucci y Curtin, 2013, p. I).

Se han identificado los rasgos del liderazgo colaborativo en contextos abiertos y relacionales, y se ha podido visualizar la tendencia del cambio de modelo de un funcionario público neutral a un facilitador de la participación y el compromiso con habilidades técnicas importantes. Esto se basa en las competencias de la colaboración, en lo interactivo y en el emprendedurismo, como fundamentales para gestionar hoy 
las redes, las iniciativas, la transparencia y rendición de cuentas, las interacciones, la colaboración, la cocreación y la participación.

Figura 4. Prerrequisito para el proceso colaborativo

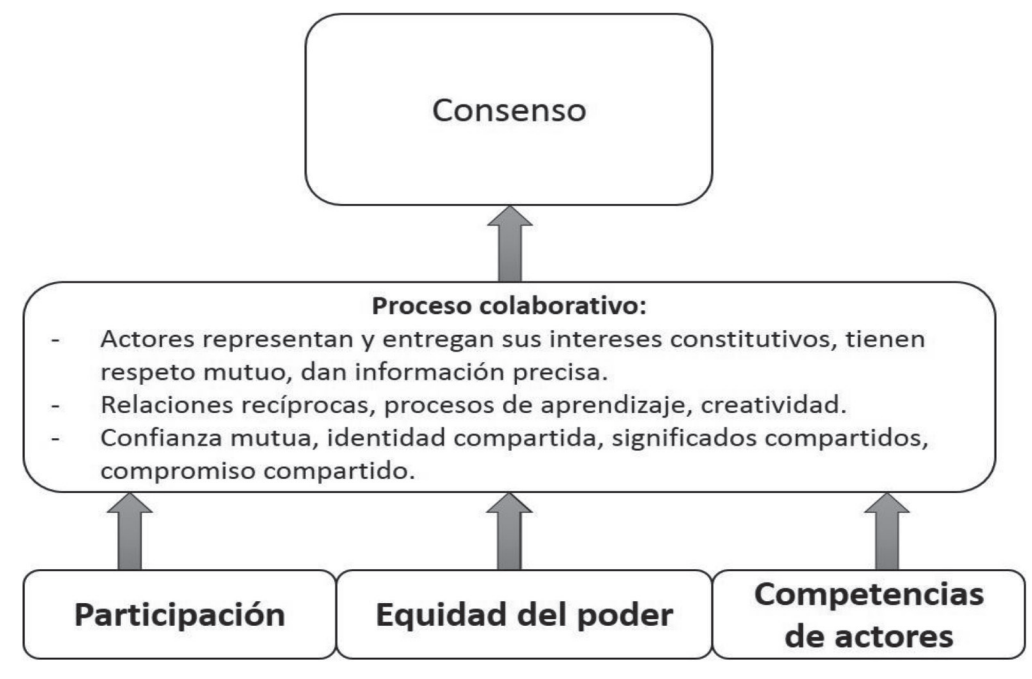

Fuente: Sufianti (2013).

\section{A manera de reflexión}

En este trabajo se han analizado los elementos operativos del concepto de gobierno abierto, por lo que se han entendido, en tanto su implementación, como cambios en los procesos, en la organización y en las formas de relación que implicarían la innovación en la parte gubernamental. Para ello, dos preguntas se respondieron: ¿qué rasgos y competencias debe tener un líder público gubernamental en los contextos de gobiernos relacionales, participativos y abiertos?, y ¿con qué saberes cívicos contarían estos líderes en su relación con los ciudadanos y los actores no gubernamentales? 
En un primer momento, se intentó poner en su lugar situacional el itinerario conceptual de la definición de gobierno abierto, para luego analizarlo en la perspectiva de una organización pública que se ha caracterizado por producir una mayor influencia compartida, consenso y confianza en el interior de toda la organización, situándola en su dimensión relacional, donde el marco de la innovación en la gestión pública está basada en saberes cívicos, en una política pública visualizada en términos de redes y en un proceso gubernamental sustentado en la gobernanza.

Así, las organizaciones públicas en las actuales circunstancias, tal como lo expresan O'Leary y Vij (2012), necesitan de gerentes públicos que trabajen colaborativamente, facilitando y operando arreglos multiorganizacionales para resolver problemas que no pueden ser resueltos -o fácilmente resueltos- por una sola organización. Es decir, esto ha sido producto de algunos cambios en el ambiente de lo público, lo privado y en las organizaciones de la sociedad civil y sin fines de lucro, que a su vez han motivado el crecimiento de lo público colaborativo.

En un segundo punto, se pudo analizar la innovación en la parte gubernamental en el marco del gobierno abierto, concibiéndola como el reconocimiento de la creatividad y el resultado que imprimen los ciudadanos y actores no gubernamentales en su relación con los funcionarios públicos. Innovar en un gobierno abierto relacional llevó a reflexionar sobre la innovación en la gestión pública, donde el centro del análisis tiene como componente los saberes cívicos, análisis que expresa que el trabajo de la gestión pública está hecho del compromiso de los funcionarios públicos y ciudadanos con el Gobierno, facilitando la inteligencia cívica, el mejoramiento del control de la gestión vía la contraloría social, así como una concepción compartida de lo público -entendida como competencias de colaboración para el logro legitimado de la eficiencia y la eficacia de los gobiernos-. 
En el último punto, dedicado a identificar los rasgos de liderazgo colaborativo en contextos abiertos, se visualizó la tendencia del paso de un funcionario público neutral a un facilitador de la participación y el compromiso con habilidades técnicas y sustantivas. Esto se basó en las competencias de la colaboración, en lo interactivo y en el emprendedurismo, como fundamentales para gestionar hoy las redes, las iniciativas, lo relacional, las interacciones, la colaboración y la participación.

Fecha de recepción: 03 de noviembre de 2014

Fecha de aceptación: 17 de agosto de 2015

Bibliografía

Aguilar Villanueva, L. F. (20I3a). Gobierno y administración pública. México: Fondo de Cultura Económica/Consejo Nacional para la Cultura y las Artes.

- (20I3b). "Challenges and Ways Forward for Public Administration Globally”, en M. S. de Vries, y G. Bouckaert (eds.), Training for Leadership (pp. 65-74). Bruselas: IIAS, IASIA, Editions Bruylant.

(2006). Gobernanza y gestión pública. México: FCE.

Agranoff, R., y McGuire, M. (2003). Collaborative public management: New strategies for local governments. Washington: Georgetown University Press.

Alford, J. (2009). Public Sector Clients: From Service-Delivery to Co-production. Basingstoke: Palgrave MacMillan.

Ashikali, T., y Groeneveld, S. (20I5). “Diversity Management in Public Organizations and Its Effect on Employees Affective Commitment Leadership and the Inclusiveness of the Organizational Cultural'. Review of Public Personnel Administration, 35(2), I46-I68.

Bailur, S., y Gigler, B. (eds.) (20।4). Closing the Feedback Loop. Can Technology Bridge the Accountability Gap? Washington: The World Bank. 
Barber, B. (2004). Democracia fuerte. Política participativa para Bibliografía una nueva época. España: Almuzara.

Bertucci, G., y Curtin, G. (20/3). The future of Connected governance in a web 2.0/3.0 World. Recuperado de: www. sspa.it/wp.../04/conclusion

Bouhadana, I., y Gilles, W. (20I4). Droit et Gouvernance des administrations publiques à l'ère du numérique. París: Les Editions IMDEV.

Brainard, L. A., y McNutt, J. G. (20I0). "Virtual GovernmentCitizen Relations: Informational, Transactional, or Collaborative?". Administration \& Society, 42(7), 836-858.

Calderón, C., y Lorenzo, S. (coords.) (2010). Open Government. Gobierno Abierto. Madrid: Algón Editores.

Castell, M. (1999). La era de la información. La sociedad red. Vol. I. España: Siglo XXI Editores.

(2008). "The New Public Sphere: Global Civil Society, Communication Networks, and Global Governance". The Annals of the American Academy of Political and Social Science, (6I6), 78-93.

Comisión Permanente de Gobierno Abierto y Transparencia (20I5). Modelo de Gobierno Abierto (working papers). México: INAI.

Cusba, E. (20I2). "Colaboración: El gobierno en doble vía con las personas", en G. Concha, y A. Nasser (coords.), El desafio en el gobierno abierto en la hora de la igualdad (PP. II5-I3I). Chile: CEPAL.

Dawes, S. S. (2010). "Stewardship and Usefulness: Policy Principles for Information-based Transparency". Governement Information Quarterly, 27(4), 377-383.

- (201 I). "Privacy and the public/private dichotomy". Revista Thesis Eleven, 107(I), I07-II5.

Denhardt, R. B., y Vinzant Denhardt, J. (2002). "The New Public Service: Serving Rather than Steering”. Public Administration Review, 60(6), 549-559. 
Bibliografía
Diario Oficial de la Federación (4 de mayo de 20I5). "Ley General de Transparencia y Acceso a la Información Pública". Recuperado de: http://www.dof.gob.mx/ nota_detalle.php?codigo $=539 \mathrm{I}$ I $43 \&$ fecha $=04 / 05 / 20$ I 5

Dryzek, J. S. (2009). "Democratization as Deliberative Capacity Building”. Comparative Political Studies, 42(I I), I379- | 402.

Fung, A. (2002). Collaboration and Countervailing Power: Making Participatory Governance Work. Harvard: JFK School of Government, Harvard University.

(2008). "Citizen Participation in Government Innovation", en S. Borins (ed.), Innovation in government. Research, Recognition, and Replication (pp. 52-70). Washington, DC: Harvard University (School of Government), Brookings Institute Press.

—, y Wright, E. O. (20I0). "En torno al gobierno participativo con poder de decisión”, en M. Canto Cha (ed.), Participación ciudadana en las políticas públicas (pp. I50-175). México: Escuela de Administración Pública del D. F., Secretaría de Educación del D. F., Siglo XXI Editores.

—, Graham, M., y Weil, D. (2007). Full Disclosure: The Perils and Promise of Transparency. Cambridge: Cambridge University Press.

Garcés Lloreda, M. T. (2006). "La Veeduría Distrital y la Política Bogotá Transparente: un modelo de control preventivo y de liderazgo ético para una gestión de integridad en el Distrito Capital", en E. Isunza Vera, y A. J. Olvera (eds.), Democratización, rendición de cuentas y sociedad civil: Participación ciudadana y control social (PP. 473-487). México: CIESAS, Universidad Veracruzana y Miguel Ángel Porrúa.

Guardián Orta, C. (2010). “¿Transparencia?”, en C. Calderón, y S. Lorenzo (coords.), Open Goverment: Gobierno abierto (pp. 75-86). Madrid: Algón Editores. 
Harrison, T. M., Guerrero, S., Burk, G. B., y Cook, M. (20I I). Bibliografía "Open Government and E-Government: Democratic Challenges from a Public Value Perspective". The Proceeding of the I 2 th Annual International Digital Governement (paper de conferencia de investigación). Workingpapers of Center for Technology in Government, University of Albany: EE. UU.

Harrison, D., y Klein, J. L. (2007). "Introduction”, en D. Harrison, y J. L. Klein (coords.), L'Innovation sociale. Émergence et effets sur la transformation des societies (Pp. I-14). Quebec: Presses de l'Université du Québec. Hevia de la Jara, F. (2006). La Contraloría Social mexicana: participación ciudadana para la rendición de cuentas. Diagnóstico actualizado a 2004. México: Centro de Investigaciones y Estudios Superiores en Antropología Social (CIESAS)/Instituto de Investigaciones Histórico-Sociales, Universidad Veracruzana.

Kelly, J., y Dodds, A. (20I2). "Public Administration in an Age of Austeruty. The future of the disciple". Public Policy and Administration, 27(3), 199-2I I.

Kickley, M. (2008). The Grounds for Citizen Engagement and The Roles of Planners. Saarbruecken: VDM.

King, C. S., y Seegers Martinelli, A. (2005). "Innovation in Citizen Engagement and Empowerment beyond Boundaries". The Innovation Journal: The Public Sector Innovation Journal, IO(I), I.

—, y Stivers, C. (eds.) (1998). Government is us. Public Administration Anti-Government Era. California: Sage Publications.

LaDuke, B. (2008). "Knowledge creation in collective intelligence”, en M. Tovey (ed.), Collective Intelligence: Creating a prosperous World at Peace (pp. 65-74). Oakton, Virginia: Earth Intelligence Network (EIN). Recuperado de: http://www.scip.org/files/resources/tovey-collectiveintelligence.pdf 
Bibliografía
Mariñez Navarro, F., y Valenzuela, R. (20I3). Gobierno abierto. ¿Más innovación?, ¿más Gobierno?, ¿más sociedad?, ¿en qué consiste? Propuesta para los gobiernos locales. México: Editorial Miguel Ángel Porrúa, EGAP.

McGuire, M. (2006). "Collaborative Public Management: Assessing What We Know and How We Know It". Public Administration Review, 66(I), 33-43.

McLean, A. (2013). Leadership \& Cultural Webs in organizations. Reino Unido: Emerald Group Publishing Limited.

Meijer, A. J., Curtin, D., y Hillebrandt, M. (20I2). "Open government: connecting vision and voice". International Review of Administrative Sciences, (78), I.

Mergel, I. (20I2). "The public Manager 2.0: Preparing the Social Media Generation for a Networked workplace". Journal of Public Affairs Education, I8(3), 467-492.

Michels, A. (20l I). "Innovations in democratic governance: how does citizen participation contribute to a better democracy?". International Review of Administrative Sciences, 77(2), 275-293.

Mothae, L., y Sindane, M. (20I3). "Leadership, Governance and Public Policy Competencies in the broader public sector", en M. S. de Vries, y G. Bouckaert (eds.), Training for Leadership (pp. 107-120). Bruselas: BruylantIASIA-IIAS.

Mulgan, G., y Albury, D. (2003). Innovation in the public sector. Londres: Cabinet Office.

Nan, T. (20I2). '“Citizens' attitudes toward Open Government and Government 2.0". International Review of Administrative Sciences, 78(2), 346-368.

Noveck, B. S. (2009). Wiki Government: How Technology Can Make Government Better, Democracy Stronger, and Citizens More Powerful. Washington: Brookings Institute Press. (2010). “The Single Point of Failure”, en D. Lathrop, y L. Ruma (eds.), Open Government. Collaboration, Trans- 
parency, and Participation in Practice. (pp. 49-70). EE. UU.: Bibliografía O'Reilly.

OCDE (20I2). La Estrategia de Innovación de la OCDE. Empezar hoy el mañana. París: OCDE-Foro Consultivo Científico y Tecnológico.

O'Leary, R., y Vij, N. (20I2). “Collaborative Public Management: Where Have We been and Where Are We Going?". The American Review of Public Administration, 42(5), 507-522.

O'Reilly, T. (2005). What is Web 2.0. Desing patterns and business models for the next generation of Software. Recuperado de: http://oreilly.com

ONU (s/f). Declaración Universal de los Derechos Humanos. Recuperado de: http://www.un.org/es/documents/udhr/ Open Government Partnership (20I I). Recuperado de: http:// www.opengovpartnership.org/

Pascual Esteve, J. M., y Tarragora Gregorió, M. (2009). Estrategia territorial y Gobierno relacional. Manual para la Planificación Estratégica de 2da. Generación. España: Junta de Andalucía, Consejería de Gobernación.

Peters, B. G. (20|4). "Implementation structures as institutions”. Public Policy and Administration, 29(2), I 3 I - | 44.

Pesch, U. (2008). "The Publicness of Public Administration”. Administration \& Society, 40(2), 170- 193.

Raelin, J. A. (20I I). “The End of Managerial Control?”. Group \& Organization Management, 36(2), I35-160.

Rainey, H. G. (2003). Understanding and Managing Public Organization. EE. UU.: Jossey-Bass Publishers.

Ramírez Alujas, Á. (20I I). "Gobierno Abierto y modernización de la Gestión Pública: tendencias actuales y el (inevitable) camino que viene. Reflexiones seminales”. Revista Enfoques, IX(I5), 99-I25.

Rizvi, G. (2008). "Innovation in Government: Serving Citizens and Strengthening Democracy", en S. Borins (ed.), Innovations in government. Research, recognition, 
Bibliografía and replication (pp. 188-198). Harvard: Ash Institute for Democratic Governance and Innovation, John F. Kennedy School of Government, Harvard University. Rosebaum, A. (20II). "The Post-Governance World: Continuing Challenges, New Opportunities”, en O. P. Dwivedi (ed.), Public Administration in Global Context: IASIA at 50 (pp. I53-180). Bruselas: Bruylant.

Saxton, G. D., y Guo, C. (20I I). “Accountability Online: Understanding the Web-Based Accountability Practices of Nonprofit Organizations". Nonprofit and Voluntary Sector Quartely, 40(2), 270-295.

Setzer, E., y Malimoud, D. (20/2). "Citizen Participation, Open Innovation, and Crowdsourcing: Challeges and Opportunities for Planning". Journal of Planning Literature, 28(I), 3-18.

Siriani, C. (2009). Investing in Democracy: Engaging Citizens in Collaborative Governance. Washington, DC: Brookings Institution Press.

Shuler, D. (2010). "Online Deliberation and Civic Intelligence", en D. Lathrop, y L. Ruma (eds.), Open Government. Collaboration, Transparency, and Participation in Practice (PP. 9I-I04). EE. UU.: O'Reilly.

(2008). "Civic intelligence and the public sphere", en M. Tovey (ed.), Collective Intelligence: Creating a Prosperous World at Peace. The Wealth of Networks: Remixed Highlights (pp. 83-94). EE. UU.: Earth Intelligence Network.

Silvia, C. (20II). "Collaborative Governance Concepts for Successful Network Leadership". State and Local Government Review, 43(I), 66-7I.

Subirats, J. (5 de abril de 20I5). "Modernidad y protagonismo ciudadano". El País. Recuperado de: www.elpais. com

Sufianti, E. (20I3). "The roles of traditional leadership in collaborative processes: Javanese Leadership Case", en 
M. S. de Vries, y G. Bouckaert (eds.), Training for LeaderBibliografía ship (Pp. I2I-I34). Bruselas: IASIA-IIAS, Éditions Bruylant. Valotti, G. (20I2). "Public sector leardership: theorical antecedents and current debate", en M. S. de Vries, y G. Bouckaert (eds.), Training for Leadership (pp. 3I-38). Bruselas: Bruylant/IASIA.

Wildrum, P. (2008). "Innovation and Entrepreneuriship in Public Services”, en P. Wildrum, y P. Koch (eds.), Innovation in Public Sector Services (pp. 3-20). Cheltenham: Edward Elgor. 